Check for updates

Cite this: RSC Adv., 2020, 10, 12680

Received 11th December 2019

Accepted 20th March 2020

DOI: 10.1039/c9ra10365a

rsc.li/rsc-advances

\section{Thymosin $\beta 4$ cytoplasmic/nuclear translocation as a new marker of cellular stress. A Caco2 case study}

\author{
Pierpaolo Coni, ${ }^{a}$ Monica Piras, ${ }^{\star a}$ Anna Mateddu, ${ }^{a}$ Marco Piludu, ${ }^{b}$ Germano Orru, ${ }^{c}$ \\ Alessandra Scano, ${ }^{\mathrm{C}}$ Tiziana Cabras, ${ }^{\mathrm{d}}$ Valentina Piras, ${ }^{\mathrm{d}}$ Joanna Izabela Lachowicz, ${ }^{a}$ \\ Mariusz Jaremko, (D) *e Gavino Faa, ${ }^{a}$ Massimo Castagnola ${ }^{f}$ and Giuseppina Pichiri ${ }^{a}$
}

Biomarkers of cell stress are important for proper diagnosis, and in studies of how cells respond to drug treatment. Biomarkers that respond early to pharmacological treatment could improve therapy by tailoring the treatment to the needs of the patient. Thymosin beta-4 $\left(T \beta_{4}\right)$ plays a significant role in many aspects of cellular metabolism because of its actin-sequestering properties. Other physiological functions of $\mathrm{T} \beta_{4}$ have been also reported. Among these, $\mathrm{T} \beta_{4}$ may play a crucial role during cellular stress. We addressed the relevance of $\mathrm{T} \beta_{4}$ in cellular stress conditions by using different treatments (serum starvation, DMSO, and butyrate administration) in a colon adenocarcinoma cell line (CaCo2), a cell line frequently used for in vitro experimental studies of $T \beta_{4}$. In this study, different stress stimuli were analyzed and the obtained results were compared using immunocytochemistry, and molecular and biochemical methods. Taken together, the data clearly indicate that the $T \beta_{4}$ peptide is involved in adaptive and defensive cellular mechanisms, and that different stress inducers lead to a similar $\mathrm{T} \beta_{4}$ cytoplasmic/nuclear translocation. The translocation of $\mathrm{T} \beta_{4}$ between the cytoplasm and the nucleus of the cell seems characteristic of a possible molecular response to cellular stress exerted by this peptide.

\section{Introduction}

Heat shock protein-72 (HSP72), fibroblast growth factor-21 (FGF21), CD133 (prominin-1), vascular endothelial growth factor, and numerous cytokines (e.g. MIP-1 $\alpha$, IL-8, MIP-1 $\beta$, resistin, GDF-15, HGF, CA125, FLRG, VCAM-1, DKK-3, sTNF-R1, CTACK, Acrp30, CXCL-16 and LYVE-1) have been identified as cancer biomarkers expressed in cells under stress conditions. ${ }^{1}$ Other biomarkers of stress conditions are still under intensive development due to their usefulness in quick diagnosis of the pathology.

Thymosin beta- $4\left(\mathrm{~T} \beta_{4}\right)$ is a 43 amino acid long, ubiquitous intracellular peptide, well known as G-actin sequestering factor in the cytoplasm of mammalian cells. ${ }^{2-4}$ Various studies suggested that this small peptide has other biological functions related to cell proliferation and differentiation that are necessary to promote and modulate wound healing and inflammatory responses. ${ }^{5}$

\footnotetext{
${ }^{a}$ Department of Medical Sciences and Public Health, University of Cagliari, Cagliari, Italy. E-mail: monipiras@hotmail.com

${ }^{b}$ Department of Biomedical Sciences, University of Cagliari, Cagliari, Italy ${ }^{c}$ Department of Surgical Sciences, OBL, University of Cagliari, Cagliari, Italy ${ }^{d}$ Department of Life and Environmental Sciences, University of Cagliari, Cagliari, Italy ${ }^{e}$ King Abdullah University of Science and Technology (KAUST), Biological and Environmental Science \& Engineering (BESE), 23955-6900 Thuwal, Saudi Arabia. E-mail: mariusz.jaremko@kaust.edu.sa

${ }^{f}$ Laboratorio di Proteomica e Metabolomica, IRCCS Fondazione Santa Lucia, Italy
}

Comparative studies on $\mathrm{T} \beta_{4}$ expression in fetal and adult human tissues showed a preferential expression of $\mathrm{T} \beta_{4}$ during fetal life. ${ }^{6}$ The role of this peptide in cardiac tissue stress and repair processes, as evidenced by epicardial cell migration, neovascularization and/or revascularization and activation of cardiac progenitor cells in the heart, is already well documented. In human corneal epithelial cells and conjunctival cells, an apoptotic $\mathrm{T} \beta_{4}$ protective effect was reported. This effect took place after stress injury induced by ethanol and hydrogen peroxide $\left(\mathrm{H}_{2} \mathrm{O}_{2}\right)$. T $\beta_{4}$ also regulates induced-proinflammatory cytokine, while blocking RelA/p65 nuclear translocation. ${ }^{7}$ Because of this, $\mathrm{T} \beta_{4}$ could be used as a new and early biomarker to diagnose pathology more quickly than cytokines.

Localization of $\mathrm{T} \beta_{4}$ may be easily altered according to different external stimuli. It can cross the nuclear membrane and act as a transcription factor in the nucleus. ${ }^{8} \mathrm{~T} \beta_{4}$ translocation was first described in a paper in which the authors injected this peptide into Xenopus laevis oocytes, where this molecule has reached both nuclear and cytoplasmic compartments. ${ }^{9}$ In IEC- 6 cells, $\mathrm{T} \beta_{4}$ was found predominantly in the nuclei and in the nuclear membrane after cell depletion of polyamines induced by alpha-difluoromethylornithine treatment. ${ }^{10}$ Thymosin $\beta 4$ has various functional roles in normal cell biology and its mechanism of action has recently been studied in various tumors. In particular, its role in colorectal cancer invasion and metastasis was suggested in several studies using in vivo and in vitro techniques. 
Nuclear localization of $\mathrm{T} \beta_{4}$ in human mammary carcinoma MCF-7 cell line was also reported. The authors, who described this phenomenon, suggested that $\mathrm{T} \beta_{4}$ enters the nucleus through an active transport mechanism, and requires a currently unidentified soluble cytoplasmic factor. The unidentified factor could be the MLH1 protein, a key enzyme in DNA mismatch repair with several additional functions, together with the intranuclear transport of $\mathrm{T} \beta_{4} \cdot{ }^{11} \mathrm{~A}$ passive, but regulated diffusion mechanism has also been proposed as another way that $\mathrm{T} \beta_{4}$ translocates into the nucleus. ${ }^{\mathbf{1 2}}$ Recently, the metal binding ability of $\mathrm{T} \beta_{4}$ was suggested as a driving force in the cellular translocation and actin binding activity of this peptide. $^{13}$ In other experiments, involving stress stimuli induced by the in vitro serum starvation in HepG2 cells, the translocation from the cytoplasm to the nucleus was reported as the characteristic signal of a possible stress regulatory function exerted by this peptide. Altogether, these data underline the ability of $\mathrm{T} \beta_{4}$ to change its subcellular localization in distinct cellular conditions of varying difficulty.

Several questions concerning $\mathrm{T} \beta_{4}$ translocation and its importance in cellular function have yet to be answered, while stress induced events can often trigger a plethora of divergent responses that may interfere with the experimental results, and with the subsequent conclusions. ${ }^{\mathbf{1 4}}$ For example, short or prolonged incubation in serum-free medium could lead uniform changes in the basal cellular activity, or it could lead to different signaling pathways showing opposing results.

In order to understand better the $\mathrm{T} \beta_{4}$ 's translocation under stress conditions, we use different stress treatments in $\mathrm{CacO} 2$ cell line, a commercial colon cancer cell line frequently used as a normal and human disease model.

\section{Material and methods}

\subsection{Cell culture}

Commercial human cell lines (CaCo2, ICLC, Genova) derived from a colon adenocarcinoma were used. CaCo2 cells were maintained in culture with MEM medium (EBSS) containing FBS (fetal bovine serum) at 10\%, 100 units per ml penicillin, $100 \mathrm{mg}$ per $\mathrm{ml}$ streptomycin, $2 \mathrm{mM}$ L-glutamine, $1 \%$ of nonessential amino acids. The confluent cells were detached using trypsin/EDTA as previously described (Pichiri et al., 2013; ${ }^{6}$ Piludu et al., $2015^{31}$ ) in order to obtain different experimental conditions.

\subsection{Experimental procedures}

CaCo2 cells were seeded $\left(2-3 \times 10^{4}\right.$ cells per $\left.\mathrm{cm}^{2}\right)$ in $100 \mathrm{~mm}$ culture dish with a cover slides inside and were incubated at $37{ }^{\circ} \mathrm{C}, 5 \% \mathrm{CO}_{2}$ as previously described (Pichiri et al., ${ }^{6}$ 2013; Piludu et al., $2015^{31}$ ).

Different experimental groups were obtained after $24 \mathrm{~h}$ of growth with complete medium. One group was incubated with a culture medium without FBS (starvation), while second and third groups were incubated in complete medium in the presence of butyrate (final concentration $1.5 \mu \mathrm{M})$ or with DMSO $(1 \%$ final concentration); the fourth group of cells were incubated in
FBS deprived medium and treated with butyrate (final concentration $1.5 \mu \mathrm{M}$ ). A control group was incubated in complete medium without any treatment. After 48 hours all samples were washed with phosphate buffered saline (PBS), fixed in cold acetone for 10 minutes, successively air dried for 30 minutes and used for immunocytochemistry, biochemistry, and RNA analysis.

\section{3. $T \beta_{4}$ immunocytochemistry analysis}

$\mathrm{T} \beta_{4}$ immunocytochemistry was performed as previously described in the literature. The cells were briefly rehydrated, and endogenous peroxidase activity was quenched ( $3 \mathrm{~min}$ ) by $0.3 \%$ hydrogen peroxide in methanol. Cells were incubated with $10 \%$ normal goat serum in PBS for 5 min to block non-specific binding and then incubated (30 min) with a polyclonal anti-T $\beta_{4}$ antibody (Abcam, Anti-Thymosin beta 4 antibody (ab14334); Bachem-Peninsula Lab, San Carlos, CA, USA) diluted 1 : 100 in a blocking solution. Cells were extensively washed with PBS containing $0.01 \%$ Triton X-100 and incubated with a secondary reagent (Envision kit) according to the manufacturer's instructions (Dako, Glostrup, Denmark). After additional washes with PBS, the color was developed using AEC reagent (Dako, Glostrup, Denmark); cells were counterstained with hematoxylin and mounted.

\subsection{Biochemistry analysis. Preparation of cytosolic and nuclear proteins/peptides for RP-HPLC-ESIMS analysis}

To evaluate the levels of $\mathrm{T} \beta_{4}$ in the cytosol and in the nucleus of the cells under different growing conditions (normal and stressing), subcellular fractions were prepared by the modified method of Galan et al. (Galan et al. 2011 ${ }^{32}$ ). using the commercial kit NE-PER Nucleus and Cytoplasmyc Extraction Reagents (Thermo Scientific-USA, www.thermoscientific.com/ pierce).

Cytosolic and nuclear proteins/peptides were fractionated with Amicon centrifugal filters (30 KDa cutoff) (Millipore, Billerica, MA, USA), dialyzed in $25 \mathrm{mM}$ sodium acetate at $\mathrm{pH} 4.2$ and then lyophilized. The powder was dissolved in $100 \mu \mathrm{l}$ of $0.1 \%$ aqueous trifluoroacetic acid (TFA), centrifuged at $8000 \mathrm{~g}$ for $10 \mathrm{~min}$ at $4{ }^{\circ} \mathrm{C}$, and the supernatant analysed by HPLC-ESIMS. Triplicates of the samples were prepared.

\subsection{HPLC-low-resolution ESI-MS analysis and quantification}

A Surveyor HPLC system (ThermoFisher, San Jose, CA, USA) connected to an LCQ Advantage mass spectrometer (ThermoFisher) was used for HPLC-low-resolution ESI-MS measurements. The chromatographic column was a Vydac (Hesperia, CA, USA) C8 with $5 \mathrm{~mm}$ particle diameter (column dimensions $150 \times 2.1 \mathrm{~mm})$. The eluents utilized for the chromatographic separations were: (eluent A) $0.056 \%$ aqueous TFA and (eluent B) $0.050 \%$ TFA in acetonitrile-water $80 / 20(\mathrm{v} / \mathrm{v})$. The applied gradient was linear from 0 to $54 \%$ in 39 min (linear) and from $54 \%$ to $100 \%$ in $10 \mathrm{~min}$ (linear), at a flow rate of $0.10 \mathrm{ml} \mathrm{min}^{-1}$. During the first $5 \mathrm{~min}$ of separation, eluate was discarded in order to avoid source contamination and instrument damage 
due to the high salt concentration. Mass spectra were collected every 3 milli-second in the positive ion mode. MS spray voltage was $5.0 \mathrm{kV}$ and capillary temperature was $255^{\circ} \mathrm{C}$.

$\mathrm{T} \beta_{4}$ was analysed in the Total Ion Current (TIC) chromatographic profiles of the examined samples by using the extracting ion current (XIC) procedure. The XIC search allowed to extract selectively the ion current peak generated by specific multiply-charged ions (charge) of $\mathrm{T} \beta_{4}$ : $993.80(+5), 1241.90(+4)$ and $1655.50(+3) \mathrm{m} / \mathrm{z}$. A window of $\pm 0.5 \mathrm{Da}$ was used to extract the XIC peak. Experimental average mass value $\left(M_{\text {av }}\right)$ was obtained by deconvolution of averaged ESI-MS spectra automatically performed by using MagTran 1.0 software.$^{15}$ Experimental $M_{\text {av }}$ was compared with the theoretical one available at the UniProt-KB human data-bank (http://us.expasy.org/tools) with the entry P62328. Experimental $M_{\mathrm{av}}$ and elution time were compared also with those obtained by HPLC-ESI-MS of the standard peptide (Bachem, Bubendorf, Switzerland).

The area of XIC peak was used for quantification, since it is proportional to the peptide concentration under constant analytical conditions. ${ }^{16}$ XIC peak areas were integrated by using the following peak parameters: baseline window 15; area noise factor 50; peak noise factor 50; peak height $15 \%$ and tailing factor 1.5. Estimated percentage error of the XIC procedure was $<8 \%$. The areas of XIC peaks were correlated to the concentrations expressed in $\mathrm{nM}$, after obtaining a linear regression between the known concentrations of the standard $\mathrm{T} \beta_{4}(0.005$, $0.025,0.05,0.10,0.25,0.35,0.5,0.75,1.00,1.25,1.50 \mu \mathrm{M})$ and the respective XIC peak areas obtained by HPLC-ESI-MS analysis.

Relative abundance (\%) in nuclear fraction (NF) with respect to cytosolic fraction (CF) was calculated in the three series of CaCo2 cells as follows:

$\% \mathrm{NF}=\mathrm{NF}$ XIC peak area $\times 100 /(\mathrm{NF}+\mathrm{CF}$ XIC peak areas $)$.

Statistical comparison of the $\mathrm{T} \beta_{4}$ concentration between the $\mathrm{CF}$ and NF samples in the different stress conditions, such as among the $\mathrm{T} \beta_{4} \%$ in $\mathrm{NF}$, was performed, with $5.0 \mathrm{GraphPad}$ Prism software, by the unpaired $t$-test with Welch's correction being the samples size equal, small and the variance unequal. The data were analysed also with one-way ANOVA as well as the multicomparison Tukey's test. $P$ values $<0.05$ was considered significant.

\subsection{HPLC-high-resolution ESI-MS/MS analysis}

Characterization of $\mathrm{T} \beta_{4}$ was performed on the intact peptide present in the samples by using an Ultimate 3000 Micro HPLC apparatus (Dionex, Sunnyvale, CA, USA) equipped with a FLM3000-Flow manager module and coupled to an LTQ Orbitrap XL apparatus (Thermofisher). The injection volume was $20 \mu \mathrm{l}$. The column was a Zorbax 300SB-C8 (3.5 $\mu \mathrm{m}$ particle diameter; $1.0 \times 150 \mathrm{~mm}$ ), and the eluents were: $\mathrm{A}, 0.1 \%$ aqueous formic acid solution, and $\mathrm{B}, 0.1 \%$ aqueous formic acid solution in acetonitrile/water $80: 20 \mathrm{v} / \mathrm{v}$. The applied gradient was from 5 to $55 \% \mathrm{~B}$ in $40 \mathrm{~min}$, from 55 to $100 \% \mathrm{~B}$ in $2 \mathrm{~min}$, from 100 to $5 \% \mathrm{~B}$ in $2 \mathrm{~min}$ for a total acquisition time of $61 \mathrm{~min}$ at a flow rate of 80
$1 \min ^{-1}$. The MS spectra were acquired in data-dependent mode in the $\mathrm{m} / \mathrm{z}$ range from 300 to 2000 . The three most abundant ions were selected and fragmented by using collision-induced dissociation (CID, 35\% normalized collision energy for $30 \mathrm{~ms}$, with an isolation width of $6-10 \mathrm{~m} / z$, activation $q$ of 0.25 . MS and MS/MS scans were acquired at a resolution of 60000 ). The capillary temperature was set to $250{ }^{\circ} \mathrm{C}$, and the source voltage was set to $4 \mathrm{kV}$. Data were generated by Xcalibur 2.2 SP1.48 (Thermo Fisher Scientific) using default parameters of the Xtract program for deconvolution. MS/MS data were analyzed by the Proteome Discoverer software (version 1.4.1.14, Thermo Fisher Scientific), based on SEQUEST HT cluster as the search engine against the UniProtKB human database (163, 117 entries, release 2018_02). For peptide matching, highconfidence filter settings were applied: the peptide score threshold was 3.0; the limits for FDR setting were Xcorr scores greater than 1.2 for singly charged ions, and 1.9 and 2.3 for doubly and triply charged ions, respectively. The precursor mass search tolerance was $10 \mathrm{ppm}$ and the fragment mass tolerance were 0.6 Da. Since $\mathrm{T} \beta_{4}$ is $\mathrm{N}$-terminal acetylated, the analysis was performed by using $\mathrm{N}$-terminal acetylation $(+42.011 \mathrm{Da})$ as a dynamic modification.

Peptide sequence was validated by manual inspection of the experimental fragmentation spectra against the theoretical ones generated by MS-Product software available at the ProteinProspector website (http://prospector.ucsf.edu/prospector/ mshome.htm).

The mass spectrometry data have been deposited to the ProteomeXchange Consortium (http://www.ebi.ac.uk/pride) via the PRIDE (Vizcaíno et al., $2016^{33}$ ) partner repository with the dataset identifier PXD012161.

Account details for reviewer use only: username: reviwer93637@ebi.ac.uk; password: uNEEGroJ.

\section{7. mRNA analysis}

To characterize better the different stress stimuli used in this study, the expression of the following genes at the mRNA level was evaluated by real time PCR: PRDX1: this gene encodes a member of the family peroxiredoxin (an antioxidant enzyme); $T X N$ : the protein encoded by this gene is involved in many redox reactions; VCAM: a protein involved in oxidative stress, and as an adhesion molecule in vascular cells; TRND: known as a mitochondrial tRNA useful for regulating the redox state of the cell; Hsp 70: the term HSP70 (Heat Shock Protein 70 kilodaltons) refers to a family of proteins expressed ubiquitously and important for the cellular machinery of protein folding. In fact, the HSP70 proteins help other nascent proteins achieve a definitive spatial structure and help protect the cell against stress factors such as heat; $B A X$ : is a protein of the Bcl-2 family that activates apoptosis; $\mathrm{HO}-1$ : is a protein with heme-oxygenase activity in substrates heme and non-heme. We also evaluated a possible change in the expression of $\mathrm{T} \beta_{4}$ induced by these different treatments.

The cells were immediately frozen after treatment and stored at $-80{ }^{\circ} \mathrm{C}$ until RNA extraction. The RNA was extracted with 
NorDiag ARROW RNA kit using the automated tool of the same company. Actin was used as a reference gene. ${ }^{17}$

The Real-time reverse-transcriptase PCR was performed using the Roche Light Cycler system and SYBR Green I kit amplification kit (Roche Diagnostics). The following primers (bactF $=5^{\prime}$-GCATGGGTCAGAAGG-3', b-actR $=5^{\prime}$-AGGCGTACAGGGATAG- $3^{\prime}$, tb4F $=5^{\prime}$-GGCCACTGCGCAGACCAGACT ${ }^{\prime}$, $\mathrm{tb} 4 \mathrm{R}=5^{\prime}$ CTTGATCCAACCTCTTTGCATCTTACAA-3') were designed using the sequences of the $\mathrm{T} \beta_{4}$ RNA (GenBank accession no. NM_001101) and the human beta-actin mRNA (GenBank accession no. NM_001101).

The $20 \mathrm{ml}$ final volume contained: $3 \mathrm{mM} \mathrm{MgCl}_{2}, 0.25 \mathrm{mM}$ of each primer, and $2 \mathrm{ml}$ of RNA extract. Cycling was performed using the following amplification conditions: an initial reverse transcription at $55{ }^{\circ} \mathrm{C}$ for $10 \mathrm{~min}$, denaturation at $95{ }^{\circ} \mathrm{C}$ for $30 \mathrm{~s}$ followed by 35 cycles at $95{ }^{\circ} \mathrm{C}$ for $10 \mathrm{~s}, 53{ }^{\circ} \mathrm{C}$ for $10 \mathrm{~s}$, and $72{ }^{\circ} \mathrm{C}$ for $8 \mathrm{~s}$ with subsequent melting analysis: heating to $95{ }^{\circ} \mathrm{C}$ for $20 \mathrm{~s}$, cooling to $45^{\circ} \mathrm{C}$ for $10 \mathrm{~s}$ and reheating to $95{ }^{\circ} \mathrm{C}$ at a rate of $0.2^{\circ} \mathrm{C}$ per second.

Fluorescence was detected at the end of the $81^{\circ} \mathrm{C}$ segment in PCR step (single mode) and at $45^{\circ} \mathrm{C}$ segment in the melting step (continuous mode) in the $\mathrm{F} 1$ channel. The relative gene expression was analyzed by using the 2-DDCT method. ${ }^{18}$

For each analysis, three distinct biological replicas were done, and quantitative data were expressed as a mean. Folding change values in $\mathrm{T} \beta_{4}$ gene expression relative to the beta-actin have been represented as mean + standard error.

\section{Results and discussion}

Several reports have shown that $\mathrm{T} \beta_{4}$, a multi-functional regenerative peptide, ${ }^{19}$ could be involved in different critical biological activities, including angiogenesis, ${ }^{20}$ wound healing, ${ }^{21}$ inflammatory response, ${ }^{22}$ cell migration, ${ }^{23}$ survival, and oxidative stress. ${ }^{24}$

The $\mathrm{T} \beta_{4}$ ability to change conformation and to translocate from the cytoplasm to the nucleus and back, was recently described and associated to stress induced in vitro in the HepG2 cell line by serum starvation. ${ }^{6}$ The significance of our previous studies was limited by discovering that stress condition like serum starvation, may be characterized by different responses that depend on the experimental conditions. ${ }^{\mathbf{1 4}}$

In order to clarify better the actual significance of intracellular trafficking of $\mathrm{T} \beta_{4}$, we used different stress stimuli in the Caco2 cell line. The immunocytochemistry, mass spectrometry, and molecular data obtained in this study strongly support the hypothesis that $\mathrm{T} \beta_{4}$ cytoplasmic/nuclear translocation could be a general defensive mechanism against multiple and different adverse environmental changes, and $\mathrm{T} \beta_{4}$ could be used as a new and early biomarker of cellular stress.

Starvation is able to induce stress by serum deprivation in vitro, ${ }^{25}$ while albumin (the most abundant protein of serum) is the main carrier of essential metal ions and fatty acids for cells. ${ }^{26}$ Butyrate, a short chain fatty acid produced by commensal bacteria of the human intestine, was already used to induce oxidative stress and apoptosis in $\mathrm{CaCo} 2$ cell cultures in Fauser et $a l^{27}$ Moreover, it was reported that butyrate could act as an epigenetic "switch" of the immune system. ${ }^{28}$ Butyric acid has been in fact associated with the ability to inhibit histone deacetylases (HDAC), and favors a state of acetylated histones in the cell. ${ }^{29}$ DMSO is a powerful oxidant that is often used as a solvent in various experimental tests and in NMR analysis, while its signals have low interferences, and it can dissolve a large number of substances. Many embryonic and/or tumor cell lines treated with DMSO in the culture medium are induced to mature, and the hiring of a morphological phenotype more "mature". Nevertheless, the mechanism of DMSO cellular stress action has not yet been completely deciphered.

Serum starvation, DMSO and butyrate treatment were used in our studies to induce in vitro cellular stress with different pathways.

\subsection{Morphological analysis of the cells}

In order to examine morphological changes in the cells, the Caco2 cells were grown under different stress conditions and immunoassayed with a polyclonal anti-T $\beta_{4}$ antibody. Specific $\mathrm{T} \beta_{4}$ immuno-reactivity was visualized as small roundish spots in all examined samples.

In detail, $\mathrm{T} \beta_{4}$ immunocytochemistry was performed in $\mathrm{CaCo} 2$ cell lines growing for $48 \mathrm{~h}$ with and without fetal bovine
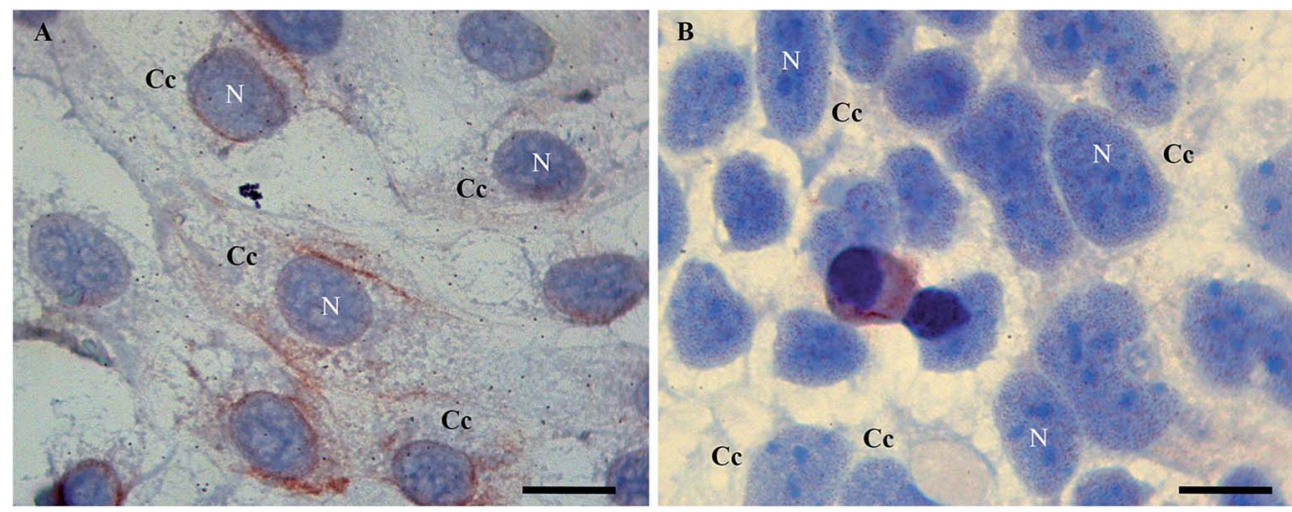

Fig. $1 \mathrm{~T} \beta_{4}$ immunoreactivity in $\mathrm{CaCo} 2$ cells cultured for $48 \mathrm{~h}$ in normal serum (A) and without serum (starvation, B). A punctate nuclear positivity is observed only in samples undergoing starvation (B) whereas in control samples nuclei are devoid of reactivity (A). $N=$ nucleus. $C=$ cytoplasmic compartment. Bars $=20 \mu \mathrm{m}$. 


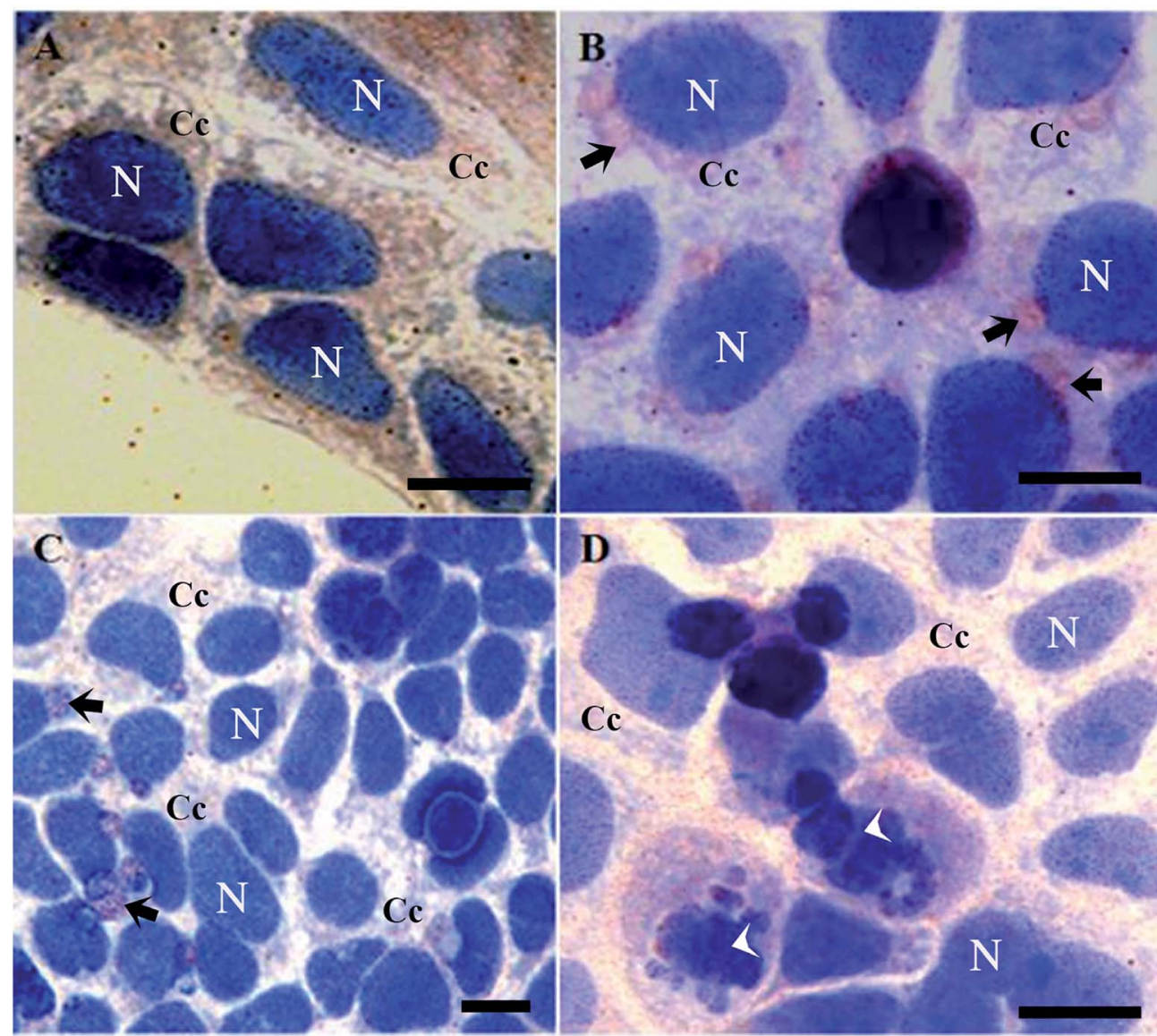

Fig. $2 \mathrm{~T} \beta_{4}$ immunoreactivity in CaCo2 cells cultured for $48 \mathrm{~h}$ with $1 \%$ of DMSO (A), $15 \mathrm{mM}$ of butyrate (B) and with $15 \mathrm{mM}$ of butyrate and starvation conditions (C and D). Butyrate and starvation conditions are characterized by the presence of several vesicles (arrows). Note the presence of apoptotic cells (arrowhead). $\mathrm{N}=$ nucleus. $\mathrm{C}=$ cytoplasmic compartment. Bars $=20 \mu \mathrm{m}$.

serum. Under normal growth conditions, the expression of $\mathrm{T} \beta_{4}$ positivity seemed to be more intense and diffuse in the cytoplasm (Fig. 1A). After $48 \mathrm{~h}$ of starvation, a pattern of punctuated nuclear $\mathrm{T} \beta_{4}$ positivity appeared (Fig. 1B).

It is worth mentioning that cells treated with butyrate presented a relevant number of $\mathrm{T} \beta_{4}$-positive vesicles (Fig. $2 \mathrm{~B}$ ).

$\mathrm{T} \beta_{4}$ translocation from cytoplasm to the nucleus in $\mathrm{CaCo} 2$ cells provoked by DMSO or butyrate treatment confirmed that $\mathrm{T} \beta_{4}$ translocation may be a general signal of cellular stress (Fig. 2A and B). Samples treated with butyrate were characterized by a relevant number of vesicles. $T \beta_{4}$ translocation and the presence of vesicles are more evident, when $\mathrm{CaCo} 2$ cells under starvation were treated with butyrate (Fig. 2C and D).

The number of cellular vesicles increased, when serum starvation and butyrate administration acted together (Fig. 2C and D), suggesting that different stimuli may exert a cumulative effect with increased stress intensity. An increased amount of vesicles could be related to an increased apoptotic activity (more $\mathrm{T} \beta_{4}$ positive vesicles and more cells showing nuclear fragmentation) (Fig. 2B), but the possible presence of $\mathrm{T} \beta_{4}$ inside these vesicles and biological significance of its presence require deeper studies. ${ }^{30}$

Taken all together, morphological studies show clearly that $\mathrm{T} \beta_{4}$ peptide is involved in adaptive and defensive cellular mechanisms, and that the cytoplasm/nuclear translocation effect, more than simply a different gene/protein expression, is related to a change in its sub cellular localization.

\subsection{Expression of $T \beta_{4}$ and other genes related to stress conditions}

$\mathrm{T} \beta_{4}$ RNA expression decreased during all our experimental treatments (Fig. 3), while other genes, related to stress

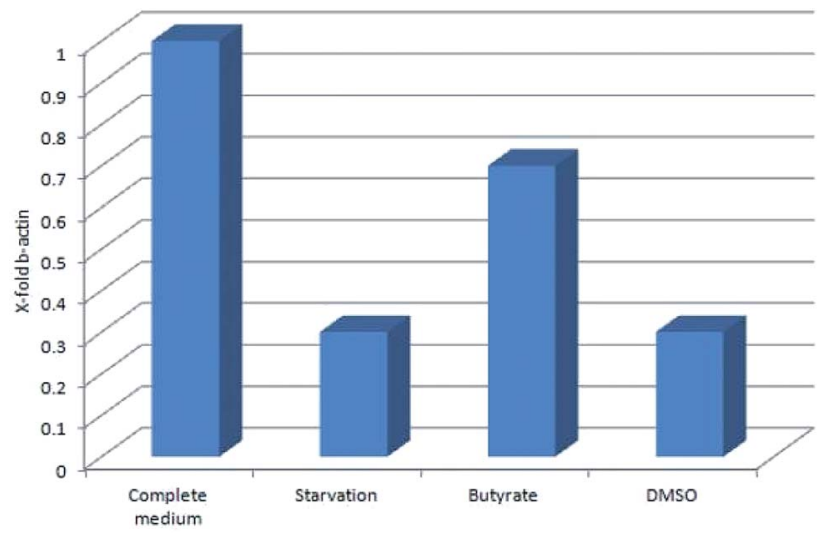

Fig. $3 \mathrm{~T} \beta_{4}$ RNA expression in CaCo2 cells exposed to different stress conditions (starvation, butyrate and DMSO). 

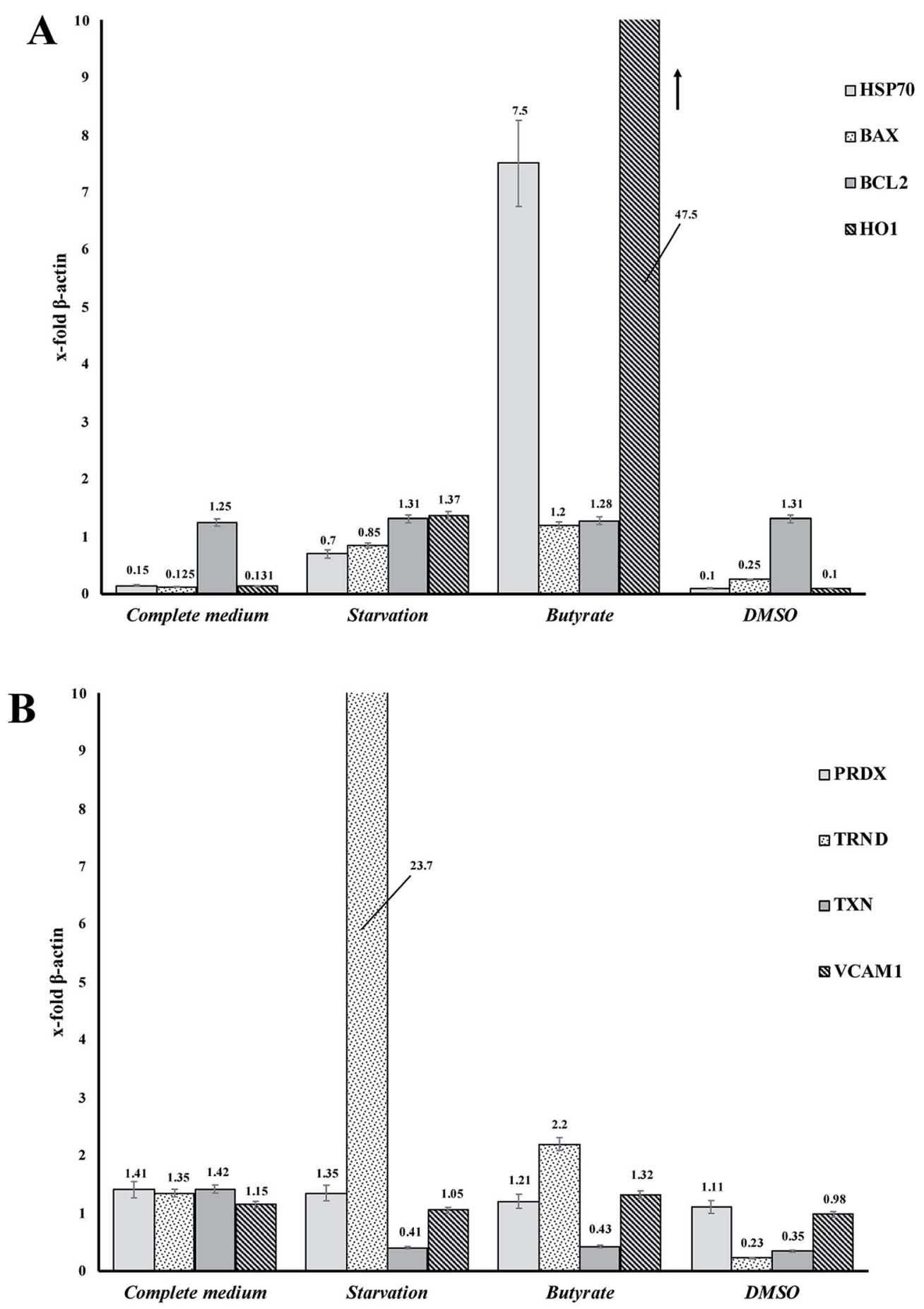

Fig. 4 (A and B) RNA expression of several genes related to stress cellular conditions in CaCo2 cells.

conditions, such as PRDX1, TXN, VCAM, TRND, Hsp 70, BAX, Bcl-2 and HO-1 showed a different pattern related to the changing conditions (Fig. 4, panels A and B).

In particular, administration of DMSO, characterized by a very low level of $\mathrm{T} \beta_{4}$ RNA, does not seem to corelate with the expression of the other examined genes, whereas butyrate treatment was followed by an increased expression of Hsp70 and HO-1, and starvation conditions induced an increased expression of TRND gene.
In fact, while the $\mathrm{T} \beta_{4}$ RNA expression in $\mathrm{CaCo} 2$ cells decreased in all the 3 different experimental stress conditions, a different mRNA pattern of several stress related genes characterized the diversity of these 3 stimuli. It can be assumed that during in vitro cellular stress induced by different stressing stimuli characterized by a different molecular and morphological expression, $\mathrm{T} \beta_{4}$ translocation may be considered a general adaptive response. 
$\mathrm{T} \beta 4$ in $\mathrm{CaC} 02$ cells

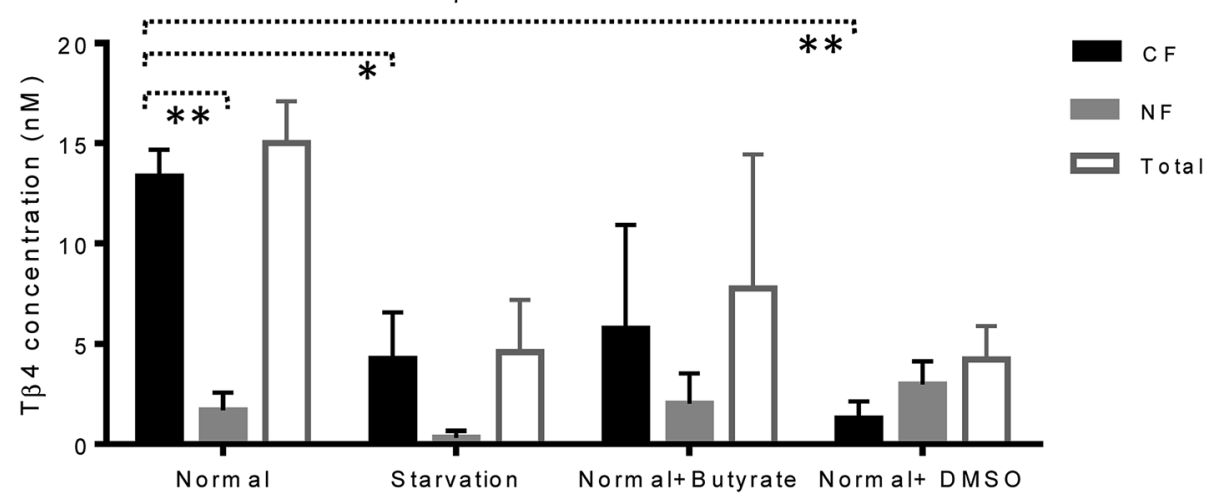

Fig. 5 Concentration (nM, mean and standard error) of $\mathrm{T} \beta_{4}$ in cytosol (black bars), and nucleus (grey bars) of CaCo2 cells under different growing conditions. The total $\mathrm{T} \beta_{4}$ levels (cytosolic + nuclear, white bars) are also reported.

\subsection{Mass spectrometry analysis}

Mass spectrometry analysis was performed in order to evaluate $\mathrm{T} \beta_{4}$ concentration changes in the cytosolic and nuclear fractions of CaCo2 cells, under normal and different stress conditions. Low-resolution liquid chromatography-mass spectrometry (LC-MS) analysis allowed detection of $\mathrm{T} \beta_{4}$ in the nuclear and cytosolic fractions at the elution time of 19.319.8 min with an experimental $M_{\text {av }}$ of $4962.8 \pm 0.6$ (theor. 4963.5 Da); the same elution time and $M_{\mathrm{av}}$ were obtained for the standard peptide analyzed in the same experimental conditions. However, the validation of $M_{\mathrm{av}}$ attribution was performed by high-resolution MS/MS spectra and the MS results were deposited on ProteomeXchange (PXD012161).

CaCo2 cells exhibited lower amounts of $\mathrm{T} \beta_{4}$ under starvation, butyrate, and DMSO treatment with respect to the normal conditions (Fig. 5). Despite the limitations due to the low

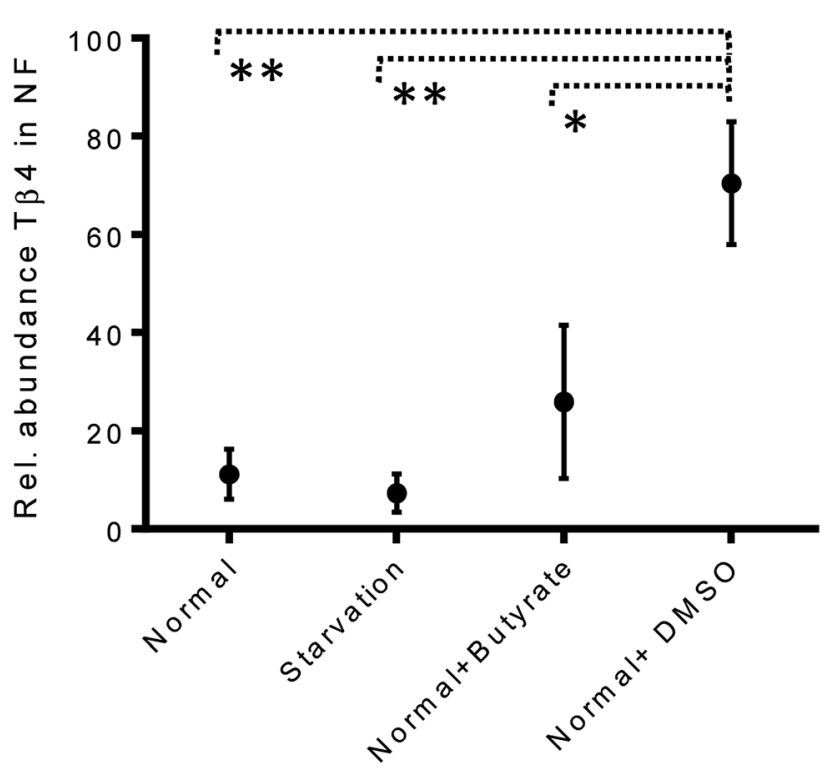

Fig. 6 Relative abundance of $\mathrm{T} \beta_{4}(\%)$ in nuclear fractions of $\mathrm{CaCo} 2$ cells under different growing conditions (from left to right: normal, starvation, normal + butyrate, normal + DMSO). number of samples under study, the statistical comparison performed between the CF and NF samples, by the $t$-test with Welch's correction, highlighted that $\mathrm{T} \beta_{4}$ concentration in normal cells was significantly higher in cytosolic than in nuclear fractions $(p<0.005)$. Moreover, cytosolic $\mathrm{T} \beta_{4}$ in starvation conditions and in presence of DMSO significantly decreased with respect to the normal conditions $(p<0.04$ and $p$ $<0.003$ respectively) (Fig. 5).

The variations of the $\mathrm{T} \beta_{4}$ concentration were comparable to that evidenced by RNA expression, and thus, appeared to be linked to a decreased $\mathrm{T} \beta_{4}$ gene expression. Among stress conditions, the trend of $\mathrm{T} \beta_{4}$ concentrations in the nuclear fraction was interesting: the minimum levels were determined under starvation and the maximum under DMSO treatment, even if not statistically different (Fig. 5). Significant variations were evidenced by ANOVA analysis of the relative abundance of $\mathrm{T} \beta_{4}$ in the nuclear fractions (Fig. 6). The average relative abundance of the peptide in the nucleus decreased from $11 \%$ in normal conditions to $7 \%$ under starvation and increased to $26 \%$ under butyrate treatment, reaching $70 \%$ in the presence of DMSO in the growing medium. The percentage of $\mathrm{T} \beta_{4}$ in NF was significantly higher in presence of DMSO with respect to the normal condition $(p<0.01)$, to the starvation $(p<0.01)$ and to butyrate presence in the medium $(p<0.05)$.

Interestingly, in the presence of DMSO, the ratio of the cytosol/nucleus fraction of $\mathrm{T} \beta_{4}$ was inverted, as showed in Fig. 5 , and since $\mathrm{T} \beta_{4}$ expression at RNA levels in DMSO-treated cells was at the very low level, nuclear $\mathrm{T} \beta_{4}$ protein concentration might be correlated only to a nuclear translocation of this protein from other cellular compartments.

Mass spectrometry analysis of the cytosolic and nuclear protein fractions, under normal and different cell stress conditions, clearly showed the differences in the two cell compartments. Interestingly, the $\mathrm{T} \beta_{4}$ protein levels observed in the nuclear fraction differed according to the varying stimuli, with minimum levels under starvation, and maximum levels after DMSO administration. DMSO represents the only condition in which $\mathrm{T} \beta_{4}$ nuclear concentration is higher than that observed in the cytosolic fraction. Given that in DMSO-treated 
cells $\mathrm{T} \beta_{4}$ RNA expression was decreased, these differences could be related to a different metabolic response or to a different cellular synchronism induced by the treatment used. In every case, the higher levels of $\mathrm{T} \beta_{4}$ peptide inside the nucleus should be interpreted as a consequence of the $T \beta_{4}$ translocation from the cytoplasm into the nuclear compartment under the stress conditions to which the cells are exposed.

\section{Conclusions}

The $\mathrm{T} \beta_{4}$ translocation process from cytoplasm to the nucleus was successfully studied in Caco 2 cells under different stress stimuli conditions. In serum starvation conditions, $\mathrm{T} \beta_{4}$ expressed the same cytoplasm/nuclear translocation in $\mathrm{Caco} 2$, as it shown in HepG2 cells in the previous studies by Pichiri et al. ${ }^{6} \mathrm{~T} \beta_{4}$ translocation in Caco 2 cells can be provoked not only by serum starvation, but also by other stress stimuli as butyrate and DMSO. Contemporary deprivation of serum and addition of butyrate to the cell growing medium lead to the higher rate of $\mathrm{T} \beta_{4}$ translocation from cytoplasmic domains to the nucleus. Overall, stress conditions lead to lower levels of $\mathrm{T} \beta_{4}$ peptide RNA expression. The immunocytochemistry, mass spectrometry, and molecular data obtained in $\mathrm{Caco} 2$ cells study, strongly support the hypothesis that $\mathrm{T} \beta_{4}$ cytoplasmic/nuclear translocation could be considered a general defensive mechanism against multiple and different adverse environmental changes. The presented experimental results together with the data that are already available in the literature demonstrate that $\mathrm{T} \beta_{4}$ could be proposed as a new and efficient biomarker of cellular stress at the early stage of pathology.

\section{Conflicts of interest}

There are no conflicts to declare.

\section{Acknowledgements}

The authors acknowledge the financial support of Fondazione Banco di Sardegna, Cagliari, Sardinia, Italy and, the financial support of Regione Autonoma Sardegna RAS-2012, LR 7 2007, CRP-60281. The funders had no role in study design, data collection and analysis, decision to publish, or preparation of the manuscript. M. J. would like to thank King Abdullah University of Science and Technology (KAUST) for the financial support. Authors would like to warmly thank Mawadda Alghrably for arranging references.

\section{References}

1 D. Cai, et al., Extensive serum biomarker analysis in patients with non-small-cell lung carcinoma, Cytokine, 2020, 126, 154868.

2 S. Nemolato, et al., Thymosin $\beta-4$ in colorectal cancer is localized predominantly at the invasion front in tumor cells undergoing epithelial mesenchymal transition, Cancer Biol. Ther., 2012, 13, 191-197.
3 M.-C. Tang, et al., Thymosin beta 4 induces colon cancer cell migration and clinical metastasis via enhancing ILK/ IQGAP1/Rac1 signal transduction pathway, Cancer Lett., 2011, 308, 162-171.

4 D. Ahmed, et al., Epigenetic and genetic features of 24 colon cancer cell lines, Oncogenesis, 2013, 2, e71.

5 T. Huff, C. S. Müller, A. M. Otto, R. Netzker and E. Hannappel, $\beta$-Thymosins, small acidic peptides with multiple functions, Int. J. Biochem. Cell Biol., 2001, 33, 205220.

6 G. Pichiri, et al., Cellular trafficking of thymosin beta- 4 in HEPG2 cells following serum starvation, PLoS One, 2013, 8, e67999.

7 P. Qiu, M. K. Wheater, Y. Qiu and G. Sosne, Thymosin $\beta 4$ inhibits TNF- $\alpha$-induced NF- $\kappa \mathrm{B}$ activation, IL-8 expression, and the sensitizing effects by its partners PINCH-1 and ILK, FASEB J., 2011, 25, 1815-1826.

8 A. L. Goldstein, E. Hannappel and H. K. Kleinman, Thymosin $\beta 4$ : actin-sequestering protein moonlights to repair injured tissues, Trends Mol. Med., 2005, 11, 421-429.

9 J. D. Watts, P. D. Cary, P. Sautiere and C. Crane RaneRobinson, Thymosins: both nuclear and cytoplasmic proteins, Eur. J. Biochem., 1990, 192, 643-651.

10 S. A. McCormack, R. M. Ray, P. M. Blanner and L. R. Johnson, Polyamine depletion alters the relationship of F-actin, G-actin, and thymosin $\beta 4$ in migrating IEC-6 cells, Am. J. Physiol.: Cell Physiol., 1999, 276, C459-C468.

11 A. Brieger, G. Plotz, S. Zeuzem and J. Trojan, Thymosin beta 4 expression and nuclear transport are regulated by hMLH1, Biochem. Biophys. Res. Commun., 2007, 364, 731-736.

12 R. E. Zoubek and E. Hannappel, Subcellular distribution of thymosin $\beta 4$, Ann. N. Y. Acad. Sci., 2007, 1112, 442-450.

13 J. I. Lachowicz, et al., Metal coordination of thymosin $\beta 4$ : Chemistry and possible implications, Coord. Chem. Rev., 2019, 396, 117-123.

14 S. Pirkmajer and A. V. Chibalin, Serum starvation: caveat emptor, Am. J. Physiol.: Cell Physiol., 2011, 301, C272-C279.

15 Z. Zhang and A. G. Marshall, A universal algorithm for fast and automated charge state deconvolution of electrospray mass-to-charge ratio spectra, J. Am. Soc. Mass Spectrom., 1998, 9, 225-233.

16 R. Inzitari, et al., HPLC-ESI-MS analysis of oral human fluids reveals that gingival crevicular fluid is the main source of oral thymosins $\beta 4$ and $\beta 10, J$. Sep. Sci., 2009, 32, 57-63.

17 J. Durzyńska and E. Barton, IGF expression in HPV-related and HPV-unrelated human cancer cells, Oncol. Rep., 2014, 32, 893-900.

18 K. J. Livak and T. D. Schmittgen, Analysis of relative gene expression data using real-time quantitative PCR and the 2- $\Delta \Delta \mathrm{CT}$ method, methods, 2001, 25, 402-408.

19 A. L. Goldstein, E. Hannappel, G. Sosne and H. K. Kleinman, Thymosin $\beta 4$ : a multi-functional regenerative peptide. Basic properties and clinical applications, Expert Opin. Biol. Ther., 2012, 12, 37-51.

$20 \mathrm{~V}$. Koutrafouri, et al., Effect of thymosin peptides on the chick chorioallantoic membrane angiogenesis model, Biochim. Biophys. Acta, Gen. Subj., 2001, 1568, 60-66. 
21 K. M. Malinda, et al., Thymosin $\beta 4$ accelerates wound healing, J. Invest. Dermatol., 1999, 113, 364-368.

22 M. Badamchian, et al., Thymosin $\beta 4$ reduces lethality and down-regulates inflammatory mediators in endotoxininduced septic shock, Int. Immunopharmacol., 2003, 3, 1225-1233.

23 M. R. Bubb, Thymosin 4 Interactions, Vitam. Horm., 2003, 66, 297.

24 S. Kumar and S. Gupta, Thymosin beta 4 prevents oxidative stress by targeting antioxidant and anti-apoptotic genes in cardiac fibroblasts, PLoS One, 2011, 6, e26912.

25 M. Aghababazadeh and M. A. Kerachian, Cell fasting: Cellular response and application of serum starvation, Journal of Nutrition, Fasting and Health, 2014, 2, 147-150.

26 S. Al-Harthi, J. I. Lachowicz, M. E. Nowakowski, M. Jaremko and $€$. Jaremko, Towards the functional high-resolution coordination chemistry of blood plasma human serum albumin, J. Inorg. Biochem., 2019, 198, 110716.

27 J. Fauser, G. Matthews, A. Cummins and G. Howarth, Induction of apoptosis by the medium-chain length fatty acid lauric acid in colon cancer cells due to induction of oxidative stress, Chemotherapy, 2013, 59, 214-224.
28 G. Serena, et al., Proinflammatory cytokine interferon- $\gamma$ and microbiome-derived metabolites dictate epigenetic switch between forkhead box protein 3 isoforms in coeliac disease, Clin. Exp. Immunol., 2017, 187, 490-506.

29 S. M. McNabney and T. M. Henagan, Short chain fatty acids in the colon and peripheral tissues: a focus on butyrate, colon cancer, obesity and insulin resistance, Nutrients, 2017, 9, 1348.

30 M. Yáñez-Mó, et al., Biological properties of extracellular vesicles and their physiological functions, J. Extracell. Vesicles, 2015, 4, 27066.

31 M. Piludu, et al., Thymosin Beta 4 May Translocate from the Cytoplasm in to the Nucleus in HepG2 Cells following Serum Starvation. An Ultrastructural Study, PLoS One, 2015, e0119642.

32 J. A. Galan, L. L. Paris, H. Zhang, J. Adler, R. L. Geahlen, et al., Proteomic Studies of Syk-Interacting Proteins Using a Novel Amine-Specific Isotope Tag and GFP Nanotrap, J. Am. Soc. Mass Spectrom., 2011, 22, 319-322.

33 J. A. Vizcaíno, et al., Nucleic Acids Res., 2016, 44(D1), D447D456. 